\title{
Multiple timing of nested intervals: Further evidence for a weighted sum of segments account
}

\author{
Donna Bryce $^{1}$ - Daniel Bratzke ${ }^{1}$
}

Published online: 17 June 2015

(C) Psychonomic Society, Inc. 2015

\begin{abstract}
How people perceive temporally overlapping intervals can inform us about the architecture and constraints of the human timing system. In the present study, we examined the time perception of two overlapping intervals in a nested context. In this context, one short interval ( $1 \mathrm{~s})$ was temporally nested within another long interval $(3 \mathrm{~s})$. The data showed that although participants' perception of the short interval was unaffected by its temporal position within the long interval, estimates of the long interval decreased, the later the short interval appeared. These data indicate that participants perceive two overlapping intervals as three segments that must be summed in order to estimate the long interval. Importantly, the temporal relationships between overlapping intervals affect the estimates, because a recency weighting is applied to each segment during the summing process. Within pacemaker-accumulator models, these results could be seen as supporting a timing system composed of a single pacemaker and a single accumulator, but they could also constrain any account of human interval timing.
\end{abstract}

Keywords Time perception · Multiple timing ·

Reproduction · Overlapping intervals .

Pacemaker-accumulator models

Donna Bryce

donna.bryce@uni-tuebingen.de

1 Department of Psychology, University of Tübingen, Schleichstrasse 4, 72076 Tübingen, Germany
Many cognitive models that aim to explain how we perceive, produce, estimate, and compare time intervals have posited that an internal pacemaker emits pulses at a certain rate (linearly or nonlinearly; Wearden \& Jones, 2007) and that these are collected by an accumulator (e.g., Treisman, 1963). The number of pulses can be stored and then used; for example, in an interval reproduction task, the participant can cease the reproduction when the same number of pulses has been accumulated as were perceived. Although these pacemakeraccumulator models of interval timing are very successful at describing how we perceive and estimate simple intervals (Rammsayer \& Ulrich, 2001), it is unclear how they can be applied to the timing of multiple overlapping intervals. In this respect, an important question is whether overlapping intervals are timed by a single pacemaker and accumulator, or whether separate pacemakers and/or accumulators are dedicated to each interval.

To investigate this question, in a previous study we asked participants to estimate two intervals of the same duration that overlapped to a lesser or greater extent (Bryce, SeifriedDübon, \& Bratzke, 2015). Predictions were derived from various timing models, defined by the number of pacemakers and accumulators available to the timing system (see also van Rijn $\&$ Taatgen, 2008). Across three experiments, the estimate of the second interval consistently increased as the intervals were more temporally separated, whereas the estimate of the first interval was either unaffected or decreased. Overall, a model constrained by a single pacemaker and a single accumulator with an additional weighted calculation feature (referred to as the $S P S A_{\text {weighted }}$ model) best explained the results. This model treats two overlapping intervals as three segments (composed of the first interval only, the overlapping part, and the second interval only), holds the representation of each segment in a memory store and then sums the relevant segments in order to estimate the two intervals, applying a recency weighting. This 
recency weighting is similar to a fading memory trace, since more recent segments receive a greater weight in the summing process than do segments perceived earlier in the sequence (as was described in Matthews, 2013). ${ }^{1}$

Although there was the most support for this model, it was also possible to predict a similar result pattern with an adapted version of a single pacemaker, multiple accumulator model (referred to as the SPMA model) in which pulses are emitted according to a linear timescale. According to this structure, the pulses emitted during each interval are collected by separate accumulators, so no summing is required, but dual-task costs are associated with having two accumulators operating simultaneously (this model was originally described by van Rijn \& Taatgen, 2008). Originally, dual-task costs were modeled as affecting the timing of both intervals; however, if the timing of only the second interval suffers from dual-task costs, then this model (referred to as the SPMA adapted model) could also predict the data pattern we observed in Bryce et al. (2015). This seems plausible if we imagine that perhaps the timing of the first interval is prioritized and not enough resources remain for the second accumulator to function adequately.

Since our previous experiments could not conclusively support one timing structure, here we present the data from a new experiment designed to further distinguish between these models. A context in which one short interval is nested within another longer interval serves this purpose well. In the present experiment, a $3 \mathrm{~s}$ interval was presented, followed by a $1 \mathrm{~s}$ interval after a stimulus onset asynchrony (SOA) of between 250 and $1,750 \mathrm{~ms}$. Thus, the short (second) interval was always completely nested within the long (first) one. The two candidate models and the adapted version of the SPMA model described above were applied to this context and predictions were derived (see Fig. 1 for an illustration, and the Appendix of Bryce et al., 2015, for details of how each model was operationalized in the previous overlapping interval context).

Descriptively, it is assumed that the SPSA $_{\text {weighted }}$ model treats the intervals in the nested context as three segments. In order to estimate the long interval, the three segments are summed and a recency weighting is applied to each segment in this summation, based on the time passed since the end of that segment. In a short SOA condition, the longest segment is the most recent, and therefore receives a full weighting; in contrast, at a long SOA the longest segment is the first in the sequence and therefore receives a low weighting. Consequently, the estimate of the long interval should decrease with increasing SOA. Since no summing is required

\footnotetext{
${ }^{1}$ It should be noted that the original weighted sum of segments model described by Matthews (2013) was agnostic about the number of pacemakers and accumulators used by the timing system. Indeed, this model can be applied to any model of human timing. However, we use this terminology so that our application of this model to the overlapping intervals context will be comparable to the other models described. This issue is further elaborated upon in the Discussion.
}

to estimate the short interval, this estimate is not affected by weighting, and would therefore be unaffected by SOA. These effects would be similar regardless of the features of the timescale (linear or nonlinear). ${ }^{2}$

If the timing system is composed of one pacemaker and separate accumulators for each interval, as in the SPMA model, the pulses emitted during each interval would be collected by the accumulators, and dual-task costs would apply during the overlapping interval (i.e., the short interval). According to the original instantiation of this model (van Rijn \& Taatgen, 2008), dual-task costs are operationalized as the accumulators being slower to update when more than one is operating simultaneously, which leads to some pulses being missed (e.g., $25 \%$ of pulses). Therefore, if pulses are emitted in a linear fashion, both estimates should be unaffected by SOA, since the number of pulses emitted during the overlapping period would be constant across SOAs. In contrast, if time is represented nonlinearly, the number of pulses emitted during the overlapping period (i.e., the short interval) would decrease with increasing SOA. Consequently, the estimate of the long interval would increase with increasing SOA, because fewer pulses would be lost due to dual-task costs. In contrast, the estimate of the short interval would decrease with increasing SOA, because the number of pulses that represent the short interval would decrease. If dual-task costs only affect the second to-be-timed interval (here, the short interval), as is suggested in the SPMA adapted model, the estimate of the long interval would be unaffected by SOA and the estimate of the short interval would decrease with increasing SOA.

In summary, whereas previous experiments with two overlapping intervals of the same duration could not decisively distinguish between the SPSA $\mathrm{A}_{\text {weighted }}$, SPMA, and SPMA $_{\text {adapted }}$ models, we expected the present nested interval context to do so. The reason for this is that in the previous overlapping interval context, the features of the models that had similar effects on time perception (recency weighting and dual-task costs) affected the estimates of both intervals, whereas in the nested interval context, recency weighting only affects the estimate of the long interval (because estimating the short interval does not require summing), and dual-task costs would affect the estimates of both intervals (according to the original SPMA model) or only the estimate of the short interval (according to the SPMA adapted model). Thus, the models

\footnotetext{
${ }^{2}$ Depending on whether the timescale is linear or nonlinear, the predicted SOA effects on the long interval differ in certain ways. That is, when there is no weighting and pulses are emitted according to a linear timescale, the long interval should be unchanged across SOAs. In contrast, when there is no weighting and pulses are emitted according to a nonlinear timescale, the long interval should be maximal when all three segments are equal $(1,000 \mathrm{~ms}$ SOA). In both cases, the recency weighting makes the long interval maximal at the shortest SOA. Thus, the predicted effect of SOA on estimates of the long interval would vary slightly depending on the linearity of the timescale (more curvilinear in a nonlinear timescale), but both would predict that estimates of the long interval would decrease as SOA increases.
} 


\section{A. Weighted single pacemaker single accumulator}

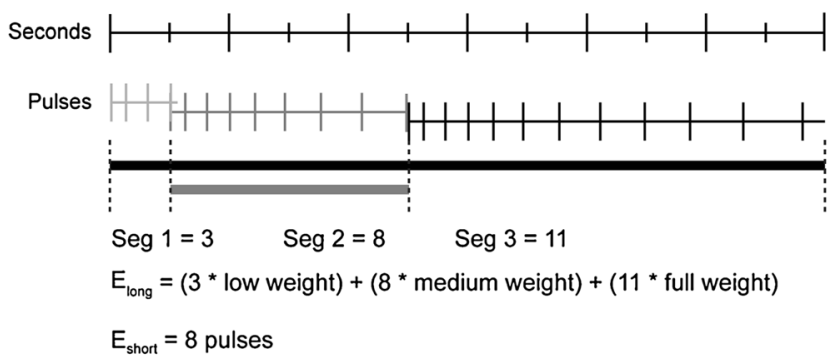

Fig. 1 Illustration of how each model (SPSA weighted $_{\text {and SPMA) would }}$ provide estimates of two intervals presented in a nested context, when a nonlinear timescale is assumed, in a trial with a short stimulus onset asynchrony. The thick black lines represent the long interval, and the thick gray lines represent the short interval. Within the nonlinear timescale, each vertical line represents one pulse, and the interpulse

can be disentangled, since they predict different effects of SOA on the estimates.

\section{Method}

\section{Participants}

Eighteen females and two males, between 19 and 33 years of age $(M=22.3$ years), participated in one $1 \mathrm{~h}$ session. The participants reported normal or corrected-to-normal vision and received either course credit or payment. Nineteen participants were right-handed, and one was left-handed. One additional participant took part in the experiment, but these data were excluded because the participant did not appear to follow the task instructions, as over $90 \%$ of the reproductions were shorter than $500 \mathrm{~ms}$.

\section{Apparatus and stimuli}

The experiment was run in a sound-attenuated, dimly illuminated room. The experiment was programmed in MATLAB using the Psychophysics Toolbox extension (Brainard, 1997), version 3.0.10, and run on an iMac with OS X. Visual stimuli were presented via the built-in LCD monitor $(60 \mathrm{~Hz})$. The stimuli were two circles with diameters of $1.75 \mathrm{~cm}$ each, presented to the left and the right of a central fixation point (square) of $0.26 \mathrm{~cm}^{2}$. The two circles were separated by a gap of $2.6 \mathrm{~cm}$. At an approximate viewing distance of $50 \mathrm{~cm}$, this resulted in visual angles of $2^{\circ}, 0.3^{\circ}$, and $3^{\circ}$ respectively. In every trial, the first presented interval had a duration of $3 \mathrm{~s}$ (referred to as the long interval), and the second presented interval had a duration of $1 \mathrm{~s}$ (referred to as the short interval).

\section{B. Single pacemaker multiple accumulators}

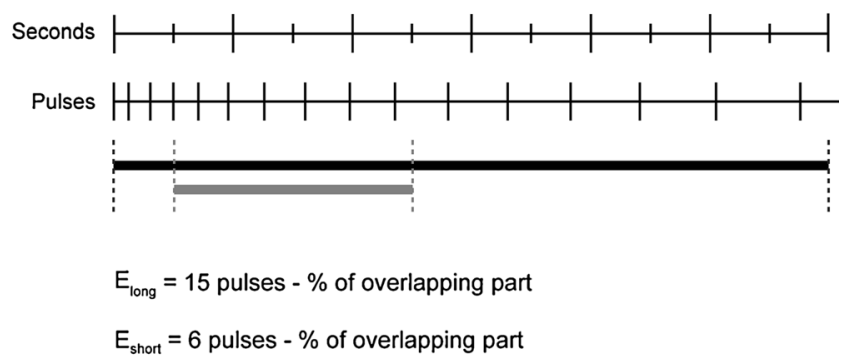

interval increases as time proceeds. In a linear timescale, the pulses would be emitted at a constant rate. The SPMA adapted model would function like the basic SPMA model, but with the dual-task costs (percent of the overlapping part) affecting only the estimate of the short interval. Note: $\mathrm{E}_{\text {long }}=$ estimate of the long interval; $\mathrm{E}_{\text {short }}=$ estimate of the short interval; Seg $=$ segment

\section{Procedure and design}

Each nested interval trial began with the presentation of a fixation point in the center of the two unfilled circles. After $1 \mathrm{~s}$, one of the circles (left or right) was filled black, and after an SOA, the other circle was also filled black. We used seven SOAs: $250,500,750,1,000,1,250,1,500$, and 1,750 ms. Each circle returned to being unfilled after $3 \mathrm{~s}$ or $1 \mathrm{~s}$, respectively. Then, $500 \mathrm{~ms}$ after the end of the long interval, estimates of the long and the short interval were collected from the participant (always in that order). In Bryce et al. (2015), both visual analog scales and reproduction were used to collect the interval estimates; the two methods produced very similar results. In this experiment, only the reproduction method was employed, since the labels of the visual analog scale might affect the estimates, especially when intervals of different durations were used. Participants were presented with a prompt, "how long was the first stimulus?" for $500 \mathrm{~ms}$, before one unfilled circle (the first one to be reproduced) was presented again in the same position on the screen (left or right). Participants gave their interval reproduction by pressing the space bar for the desired amount of time, and for the duration of the reproduction, the circle was filled black. After a $500 \mathrm{~ms}$ gap, the reproduction for the short interval was collected in the same way. Every combination of stimuli was presented once in each block, $7 \mathrm{SOA} \times$ 2 Start Sides (left or right), resulting in 14 experimental trials per block. The trials were presented in a random order. First, participants completed a practice block, and then ten experimental blocks of nested interval trials.

After ten blocks of nested interval trials, the participant completed two blocks of single interval trials. In these trials, one stimulus (a circle in the center of the screen) of either 1 or $3 \mathrm{~s}$ duration was presented. Reproductions were collected in the same way as in the nested interval trials. Each single interval was presented seven times in each block, in a random order, resulting in 14 experimental trials per block. 


\section{Analysis}

Trials in which one or both reproductions were extremely long or extremely short were not analyzed. That is, if the reproduction of the long interval was longer than 5,000 $\mathrm{ms}$ or shorter than $500 \mathrm{~ms}$, or if the reproduction of the short interval was longer than $3,000 \mathrm{~ms}$ or shorter than $250 \mathrm{~ms}$, the trial was excluded from further analyses. This accounted for 159 trials, which was $4.7 \%$ of the trials. ${ }^{3}$ The mean reproductions for the long and short intervals were analyzed in SOA (7) $\times$ Start Side (2) repeated measures analyses of variance (ANOVAs). The Greenhouse-Geisser correction was used to adjust $p$ values where appropriate. Post-hoc Tukey HSD tests were used to examine the ANOVA contrasts. Standard errors for withinsubjects designs were calculated according to Morey (2008). The mean reproductions in the single interval trials were also compared to the relevant mean reproduction in each overlapping SOA using post-hoc Tukey HSD tests. Furthermore, although the instructions to participants only emphasized precision and not speed, we reasoned that the reproduction onset time (the time taken to begin each reproduction, after the unfilled circle was presented) might provide some additional insights into how participants completed this task. Thus, the mean reproduction onset times were analyzed in the same way as the mean reproductions, described above.

\section{Results}

The mean reproductions for the long and short intervals in both the nested and single contexts can be seen in Fig. 2A and $\mathrm{B}$, with dotted horizontal lines indicating the objective interval duration. It is immediately clear that participants underestimated the $3 \mathrm{~s}$ interval, but the $1 \mathrm{~s}$ interval was relatively accurately reproduced (long $M=2,457 \mathrm{~ms}$, short $M=$ $1,014 \mathrm{~ms}$, in the nested interval context). Consistent with the SPSA $_{\text {weighted }}$ model, when one interval was nested within another, the reproductions of the long interval decreased with increasing SOA by approximately $123 \mathrm{~ms}, F(6,114)=3.66$, $p=.023$, whereas the reproductions of the short interval were unaffected by SOA, $F(6,114)=1.79, p=.160$. Post-hoc tests indicated that the long interval reproductions were significantly longer in the $500 \mathrm{~ms}$ SOA condition than in the $1,500 \mathrm{~ms}$ ( $p$ $=.01)$ and $1,750 \mathrm{~ms}$ SOA conditions $(p=.005)$. The side at which the first stimulus appeared did not affect the reproductions [long, $F(1,19)=0.21, p=.650$; short, $F(1,19)=0.40, p$ $=.532]$, nor was the Start Side $\times$ SOA interaction significant for either interval reproduction $[\operatorname{long}, F(6,114)=1.85, p=$ .095 ; short, $F(6,114)=0.80, p=.575]$.

When it was presented as a single interval, participants still underestimated the $3 \mathrm{~s}$ interval, but to a lesser degree than

\footnotetext{
${ }^{3}$ The pattern of results did not differ when outliers were not removed.
}

when it was presented in a nested context (single context $M$ $=2,616 \mathrm{~ms}$; the data point within the shaded area in Fig. 2A). Post-hoc tests indicated that the reproduction of the long interval was significantly longer in the single interval context than in the $1,250,1,500$, and 1,750 ms SOA conditions (all $p$ s $<.01)$. In contrast, the mean reproduction of the $1 \mathrm{~s}$ interval was close to the objective interval $(M=989 \mathrm{~ms})$ and did not significantly differ from the short interval reproduction in any of the nested conditions.

Mean reproduction onset times are presented in Fig. 2C and D. The mean time taken to initiate the reproduction of the long interval in the nested interval context was $465 \mathrm{~ms}$; this was unaffected by SOA, $F(6,114)=1.44, p=.243$, and start side, $F(1,19)=1.04, p=.320$, and did not differ significantly from the value in the single interval context $(M=$ $429 \mathrm{~ms}$ ). In contrast, in the nested interval context the reproduction onset time of the short interval increased with increasing SOA, $F(6,114)=6.90, p<.001$, from 489 to $650 \mathrm{~ms}$. Participants began the reproduction of the $1 \mathrm{~s}$ interval after only $473 \mathrm{~ms}$, on average, when it was presented as a single interval; this was significantly faster than in three of the nested interval conditions $(1,000,1,500$, and 1,750 ms SOA conditions; $p s<.05)$. The reproduction onset time of the short interval was unaffected by start side, $F(1,19)=0.22, p=.641$, and the Start Side $\times$ SOA interaction did not reach significance, $F(6,114)=1.54, p=.171$.

\section{Discussion}

In the present study, we examined how participants perceive two intervals presented in a nested context. Participants' reproductions of the long interval were affected by when the short interval appeared within it. That is, the later the short interval appeared, the shorter was the reproduction of the long interval. In contrast, reproductions of the short interval were unaffected by its temporal position within the long interval. These findings are entirely consistent with the SPSA $\mathrm{w}_{\text {weighted }}$ model, and inconsistent with both versions of the SPMA model. This suggests that participants perceive these intervals as three segments that must be summed in order to reproduce the long interval. Importantly, this reproduction is affected by a delay of the short interval's onset, because a recency weighting is applied to each segment during the summing process.

The only aspect of the data pattern that deviated slightly

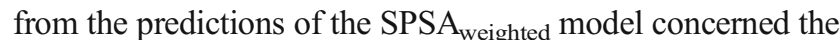
absolute values of the long interval reproduction when it was presented singly or in a nested context. That is, if the recency weighting applied to segments had a minimum value of 1 (as it was described in the original model of Matthews, 2013), the long interval would be reproduced as longer when it was presented in a nested context than when it was presented in a 
A. Mean reproductions: Long interval

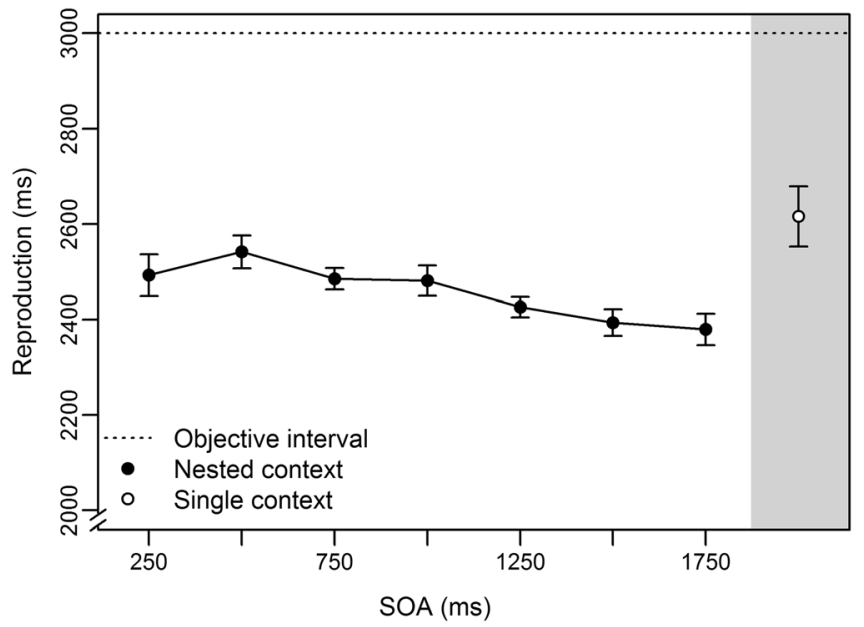

C. Reproduction onset times: Long interval

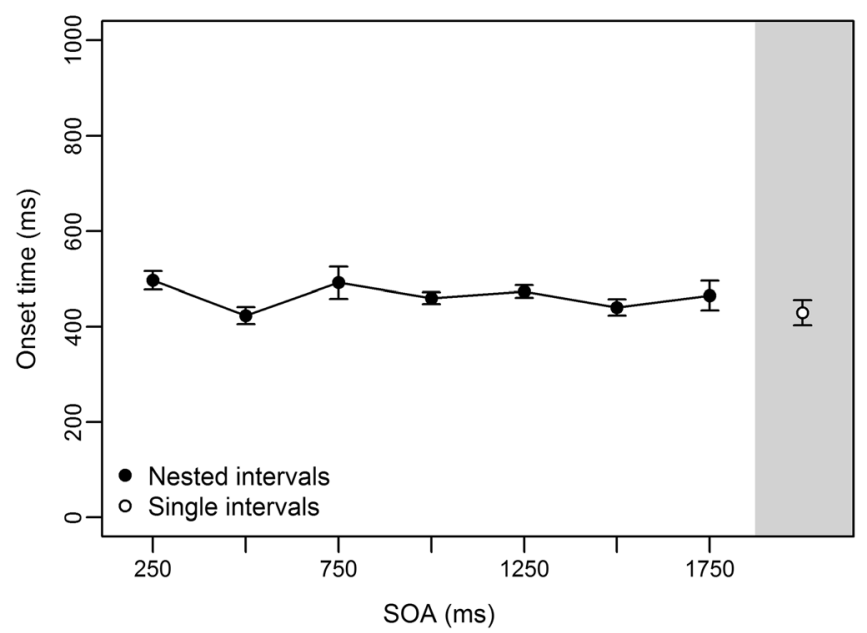

Fig. 2 (Top) Mean reproductions of the long (A) and short (B) intervals as a function of stimulus onset asynchrony (SOA), and the equivalent single interval reproductions (white circle in each gray area). The dotted lines indicate the objective interval values. Please note that the absolute values of the $y$-axes are different, but they represent the same scale.

single context. However, our data indicated that the opposite is true - reproductions were longer in the single context, that is, when no summing was required, and therefore no recency weighting was applied. This suggests that the weighting parameter should perhaps be altered to better explain our data. It is plausible that the parameter values of the weighted sum of segments model would vary between the experiments reported in Matthews (2013) and our own experiments (present study and Bryce et al., 2015), since there were various differences between the timing contexts investigated.

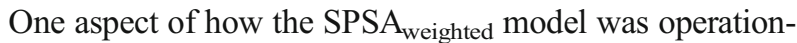
alized for the present timing context could be questionedthat the short interval was not subject to any recency weighting. It could be argued that the representation of the short interval would also be weighted according to the time
B. Mean reproductions: Short interval

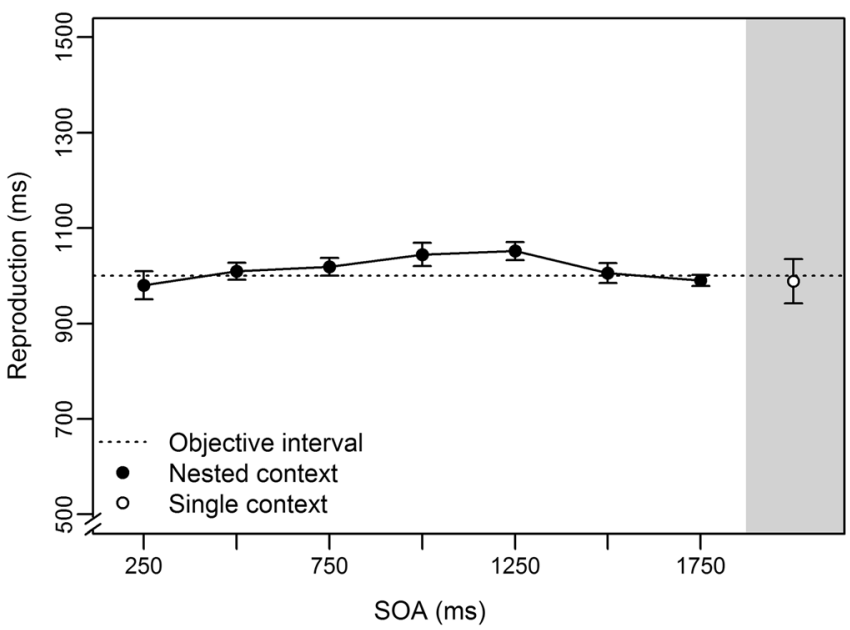

D. Reproduction onset times: Short interval

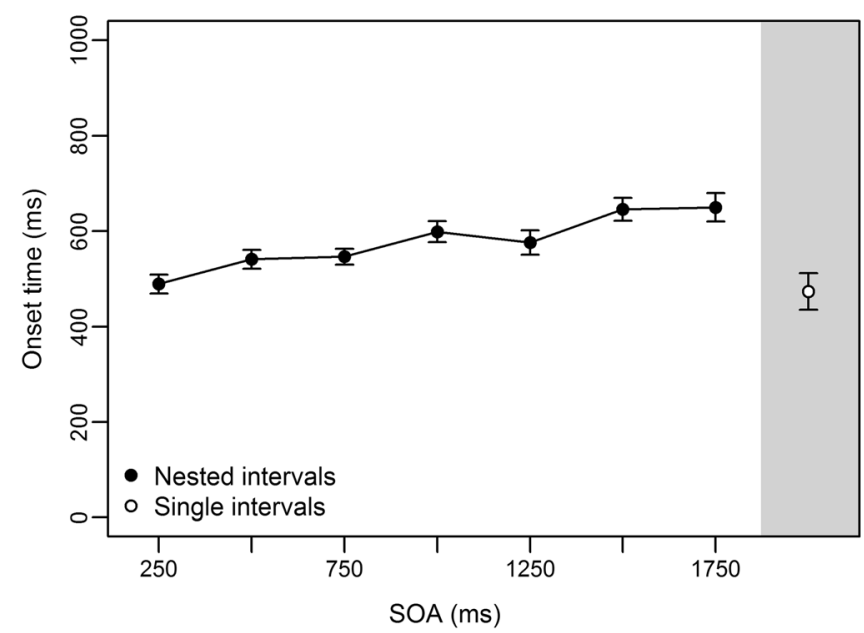

(Bottom) Mean reproduction onset times for the long (C) and short (D) intervals as a function of SOA, and the equivalent onset times for single interval reproductions (white circle in each gray area). Error bars represent within-subjects standard errors

that had passed since the end of that segment. That would mean that the earlier the short interval was presented (shortSOA conditions), the shorter that interval would be perceived. The likelihood of this depends on what the recency weighting represents psychologically. If the recency weighting occurs because pulses are lost from the representation of each segment as time progresses, a weighting should indeed be applied to the short interval; if the recency weighting is due to an error in the calculation process, it should not be. Empirically, we have two pieces of evidence supporting the latter suggestion that a weighting is only applied when summing is required. First, in Bryce et al. (2015) the two intervals were also presented in a non-overlapping context (analogous to "single" interval trials), in one trial with a gap of either 0 or $250 \mathrm{~ms}$ between them. In this case, the first interval ended either 2,000 
or 2,250 ms before the end of the trial. Thus, if a recency weighting was also applied to the estimate of this interval, it would be shorter than in all overlapping conditions (since more time had passed after the end of the segment). However, estimates of the first interval were longer in nonoverlapping than in overlapping conditions. Second, in the nested interval context of the present experiment, if a recency weighting was also applied to the short interval, the reproduction of this interval should increase with increasing SOA. Furthermore, the short interval reproduction in the single interval context should be most similar to the longest reproduction of the $1 \mathrm{~s}$ interval in the nested context (i.e., in the $1,750 \mathrm{~ms} \mathrm{SOA}$ ). Instead, we observed no SOA effect on the reproductions of the short interval. Thus, it seems likely that the recency weighting is only applied when two or more segments must be summed, as was originally proposed by Matthews (2013).

Additionally, an analysis of the time taken to begin each reproduction gives us an insight into the strategy used by participants to estimate nested intervals. That is, the longer was the SOA, the later participants started the reproduction of the short interval. This suggests that participants "replayed" the whole sequence to themselves even when they were asked to provide only one reproduction. This finding could be interpreted as further evidence that participants do not treat the two intervals as two separable, independent intervals, but as component parts of one sequence. Since we used a fixed intertrial interval, it is impossible to determine whether this increase in reproduction onset times with SOA represents the interval from the start of the trial to the onset of the short interval or that from the start of the long interval to the start of the short interval (i.e., the SOA). This issue could be addressed by introducing a variable intertrial interval; if the reproduction onset time for the long interval increased with increasing intertrial interval, we could conclude that participants "replay" the whole trial, and not just the SOA.

This report began with the question of whether overlapping intervals are timed by a single pacemaker and accumulator, or whether separate pacemakers and/or accumulators are dedicated to each interval. The model that we described as having a single pacemaker and single accumulator structure with an additional recency weighting constraint can account for the present data pattern. However, whether this therefore means that the timing system is constrained by only one pacemaker and one accumulator should be considered further. We posit that this approach (timing individual segments separately and then summing them accordingly) does strongly support the constraint of a single pacemaker, since it would be more efficient for the timing system to dedicate separate pacemakers to each interval, if this were possible, than to complete an additional calculation. What is less clear is what this approach says about the number of accumulators. Our application of the SPSA $_{\text {weighted }}$ model implicitly assumes that the representation of each segment (i.e., the number of pulses collected by the accumulator) can be stored in some long-term store until the representations are used in the summation. Additionally, we assume that the memory store is separate from the accumulator, an assumption consistent with how the memory store is typically depicted in pacemaker-accumulator models (see, e.g., Church, 1984). However, if the memory store is integrated within the accumulator, we would in fact need there to be multiple accumulators in the timing system to make the weighted sums model possible. The reason that this was not originally modeled as an option is that van Rijn and Taatgen (2008) posited that multiple pacemakers cannot operate simultaneously without dual-task costs (i.e., pulses being lost). If we reject this assumption, a single pacemaker, multiple accumulator model without dual-task costs and with an additional recency weighting in the summation could also account for our observed data patterns. Indeed, although pacemaker-accumulator models provide a useful framework for timing and are the most popular of the current timing models, there are alternative models (e.g., Jones \& Boltz, 1989; Staddon \& Higa, 1996; Wackermann \& Ehm, 2006). In fact, we posit that the approach described here could in fact constrain any model of human interval timing.

Regardless of whether the timing system has a single or multiple accumulators, the present study provides strong evidence that each segment is timed separately and that these representations are summed (with a recency weighting) in order to estimate overlapping intervals. Nevertheless, some questions remain open with regard to the generalizability of this model. For instance, does this approach hold true when additional overlapping intervals are to be timed, or when the intervals are represented by different modalities? With regard to the former question, we hypothesize that the $\mathrm{SPSA}_{\text {weighted }}$ model would successfully describe how people time more than two overlapping intervals, because Matthews (2013) found that it accounted for the timing of sequences composed of up to six segments. With regard to the latter question, the hypotheses are less clear. Although it has been suggested within pacemaker-accumulator models that pulses may be emitted at different rates for timing auditory and visual stimuli (Wearden, Edwards, Fakhri, \& Percival, 1998), we found that the SPSA $\mathrm{w}_{\text {weighted }}$ model successfully described the data when intervals of different modalities overlapped to varying degrees (Exp. 2 in Bryce et al., 2015). However, the results also indicated that the order in which the modalities were presented had an impact on how they were perceived. Since the SPSA $_{\text {weighted }}$ model as it is currently described cannot account for this effect, this is an interesting avenue for future research.

We can fairly confidently generalize across timing methods, since we have found evidence of similar effects across reproduction and visual analog scales (Bryce et al., 
2015). Furthermore, we have reason to believe that this model can also explain previous results obtained using the method of interval production (when participants must repeatedly produce a previously learned interval). That is, the only other attempt to examine the timing of two intervals that overlapped to a lesser or greater extent did so using an interval production task (van Rijn \& Taatgen, 2008). In this task, participants prelearned a $2 \mathrm{~s}$ interval and had to produce two such intervals in an overlapping context (the onset of the second interval was controlled by the experimenters, and delaying this onset resulted in the intervals having a smaller overlap). These authors concluded that their data supported a single pacemaker, single accumulator model, and that pulses were emitted nonlinearly. Importantly, the nonlinear timescale was responsible for the SOA effects on the second interval estimate. However, as we described in our previous article, a single pacemaker, single accumulator model with an additional recency weighting in the summation could also explain their results (though we do not comment on the linearity of the timescale). Although at the moment there are no empirical data on interval production in a nested context, the reproduction onset times observed in the present study suggest that participants could be providing their reproductions as if they had to produce each interval sequentially (i.e., "replaying" the SOA before providing the short interval reproduction). Thus, there is no evidence that different strategies would be used depending on the method (reproduction vs. production) employed.

In conclusion, the present experiment was conducted with the aim of distinguishing between two candidate models for the time perception of overlapping intervals. The results clear-

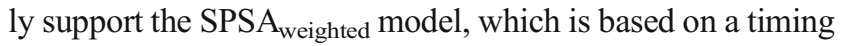
architecture with a single pacemaker and (most likely) a single accumulator. These constraints force participants to perform a calculation to estimate overlapping intervals. Importantly, this calculation is not error-free, since the more time that passes after the end of a segment, the less the segment contributes to the final estimate. Whether this recency weighting is a specific feature of the timing system or a more general memory limitation remains an open question.
Author Note Thanks to Anna-Lena Kraft for assistance with the data collection. This research was supported by the Deutsche Forschungsgemeinschaft (Grant No. BR 4297/1-1).

\section{References}

Brainard, D. H. (1997). The Psychophysics Toolbox. Spatial Vision, 10, 433-436. doi:10.1163/156856897X00357

Bryce, D., Seifried-Dübon, T., \& Bratzke, D. (2015). How are overlapping time intervals perceived? Evidence for a weighted sum of segments model. Acta Psychologica, 156, 83-95. doi:10.1016/j.actpsy. 2014.12.007

Church, R. M. (1984). Properties of the internal clock. Annals of the New York Academy of Sciences, 423, 566-582. doi:10.1111/j.1749-6632. 1984.tb23459.x

Jones, M. R., \& Boltz, M. (1989). Dynamic attending and responses to time. Psychological Review, 96, 459-491. doi:10.1037/0033-295X. 96.3.459

Matthews, W. J. (2013). How does sequence structure affect the judgment of time? Exploring a weighted sum of segments model. Cognitive Psychology, 66, 259-282. doi:10.1016/j.cogpsych.2013.01.001

Morey, R. D. (2008). Confidence intervals from normalized data: A correction to Cousineau (2005). Tutorials in Quantitative Methods for Psychology, 4, 61-64.

Rammsayer, T., \& Ulrich, R. (2001). Counting models of temporal discrimination. Psychonomic Bulletin \& Review, 8, 270-277. doi:10. 3758/BF03196161

Staddon, J. E. R., \& Higa, J. J. (1996). Multiple time scales in simple habituation. Psychological Review, 103, 720-733. doi:10.1037/ 0033-295X.103.4.720

Treisman, M. (1963). Temporal discrimination and the indifference interval: Implications for a model of the "internal clock". Psychological Monographs: General and Applied, 77(13, Whole No. 576), 1-31. doi:10.1037/h0093864

van Rijn, H., \& Taatgen, N. A. (2008). Timing of multiple overlapping intervals: How many clocks do we have? Acta Psychologica, 129, 365-375. doi:10.1016/j.actpsy.2008.09.002

Wackermann, J., \& Ehm, W. (2006). The dual klepsydra model of internal time representation and time reproduction. Journal of Theoretical Biology, 239, 482-493. doi:10.1016/j.jtbi.2005.08.024

Wearden, J. H., Edwards, H., Fakhri, M., \& Percival, A. (1998). Why "sounds are judged longer than lights": Application of a model of the internal clock in humans. Quarterly Journal of Experimental Psychology, 51B, 97-120. doi:10.1080/713932672

Wearden, J. H., \& Jones, L. A. (2007). Is the growth of subjective time in humans a linear or nonlinear function of real time? Quarterly Journal of Experimental Psychology, 60, 1289-1302. doi:10.1080/ 17470210600971576 